\title{
The coincidence index for fundamentally contractible multivalued maps with nonconvex values
}

\author{
by Dorota Gabor (Toruń)
}

\begin{abstract}
We study a coincidence problem of the form $A(x) \in \phi(x)$, where $A$ is a linear Fredholm operator with nonnegative index between Banach spaces and $\phi$ is a multivalued $A$-fundamentally contractible map (in particular, it is not necessarily compact). The main tool is a coincidence index, which becomes the well known Leray-Schauder fixed point index when $A=$ id and $\phi$ is a compact singlevalued map. An application to boundary value problems for differential equations in Banach spaces is given.
\end{abstract}

Introduction. When studying boundary value problems for differential equations or inclusions one usually encounters the question of solvability of an inclusion

$$
N(x) \in G(x),
$$

where $N: X \rightarrow Y$ is a bounded linear operator between Banach spaces and $G: \operatorname{cl} U \rightarrow Y$ is a single- or multivalued transformation defined on the closure of an open set $U \subset X$, subject to some "boundary" conditions of the form

$$
l(x) \in B(x),
$$

where $l: X \rightarrow E^{\prime}$ is a bounded linear map into a Banach space $E^{\prime}$ and $B: \operatorname{cl} U \rightarrow E^{\prime}$ is again a single- or multivalued transformation.

For instance, let $C=C^{1}([0, T], E)$ be the space of $C^{1}$-functions defined on the interval $[0, T]$ with values in a Banach space $E$ and let $f:[0, T] \times$ $E \times E \rightarrow E$ be a continuous map. If we study the existence of solutions to the general boundary value problem

$$
\left\{\begin{array}{l}
u^{\prime}(t)=f\left(t, u(t), u^{\prime}(t)\right) \\
l_{1}(u(0))+l_{2}(u(T)) \in b(u)
\end{array}\right.
$$

2000 Mathematics Subject Classification: 55M20, 34G20, 47H11, 34B15.

Key words and phrases: boundary value problem in Banach spaces, Fredholm operator, coincidence points, fixed point index, condensing map. 
where $l_{1}, l_{2}: E \rightarrow E^{\prime}$ are bounded linear maps, and $b: C \multimap E^{\prime}$ is a multivalued map ( $E^{\prime}$ is a Banach space), then we reformulate it as

$$
\left\{\begin{array}{l}
y(t)=f\left(t, z+\int_{0}^{t} y(s) d s, y(t)\right) \\
l_{1}(z)+l_{2}\left(z+\int_{0}^{T} y(s) d s\right) \in b\left(z+\int_{0} y(s) d s\right) .
\end{array}\right.
$$

Obviously, if $(z, y) \in E \times C([0, T], E)$ is a solution to $(4)$, then $u(t)=$ $z+\int_{0}^{t} y(s) d s$ is a solution to (3).

If we set

$$
\begin{gathered}
x=(z, y), \quad N(z, y)=y, \\
G(z, y)=f\left(\cdot, z+\int_{0}^{\dot{1}} y(s) d s, y(\cdot)\right), \quad l(z, y)=l_{1}(z)+l_{2}(z), \\
B(z, y)=b\left(z+\int_{0}^{1} y(s) d s\right)-l_{2}\left(\int_{0}^{T} y(s) d s\right),
\end{gathered}
$$

then problem (4) is equivalent to (1), (2).

Observe that in case $E=E^{\prime}, l_{1}=\mathrm{id}_{E}, l_{2}=-\mathrm{id}_{E}$ and $b \equiv 0,(3)$ becomes an ordinary periodic boundary value problem.

It is clear that without suitable assumptions concerning $N, G, l$ and $B$ it does not make sense to study the solvability of (1), (2). To see what type of hypotheses are most reasonable, let us further transform the problem considered. Namely, putting $A=(N, l): X \rightarrow Y \oplus E^{\prime}$ and $\phi=(G, B)$ : $\operatorname{cl} U \multimap Y \oplus E^{\prime}$, we arrive at a coincidence problem (a generalized fixed point problem) of the form

$$
A(x) \in \phi(x) .
$$

Problems of this type have been intensively studied by many authors, especially when $\phi$ is a compact map and $A$ is the identity (the Leray-Schauder fixed point theory) or $A$ is a Fredholm operator of index 0 (e.g. Mawhin [17], Pruszko [18]) or of nonnegative index (Kryszewski [12]). However, boundary value problems of the form (3) translated into the form (5) rarely lead to a compact map $\phi$. Hence, there is a need to develop a framework to study problem (5) also in the case when $A$ is a Fredholm operator of nonnegative index and $\phi$ belongs to a more general class of nonlinear (single- or multivalued) transformations, e.g. condensing, ultimately compact or fundamentally contractible maps.

The paper is organized as follows. At the end of this section we introduce some notions and definitions. In Section 1 we describe $u$-fundamentally contractible maps and give some examples. Section 2 is technical-it prepares 
tools for Section 3, which is devoted to the construction of a coincidence index in the class of maps considered. Finally, in Section 4 we present an abstract existence result and illustrate it by a boundary value problem in Banach spaces.

All topological spaces considered are metric, and all singlevalued maps are continuous.

Let $E, E^{\prime}$ be Banach spaces with norms $\|\cdot\|_{E},\|\cdot\|_{E^{\prime}}$, respectively, and let $X \subset E, Y \subset E^{\prime}$. By a multivalued map $\varphi$ from $X$ to $Y$ (denoted by $\varphi: X \multimap Y$ ) we understand an upper semicontinuous transformation which assigns to any $x \in X$ a nonempty compact set $\varphi(x) \subset Y$. Recall that $\varphi$ is upper semicontinuous (u.s.c.) if, for every closed $B \subset Y$,

$$
\varphi^{-1}(B)=\{x \in X: \varphi(x) \cap B \neq \emptyset\}
$$

is closed in $X$. As a general reference for multivalued maps we suggest [8] or $[13]$.

Throughout the paper we use the following notation: if $V$ is a subset of a Banach space $E$, then by $\mathrm{cl} V$ we denote the closure of $V$, by bd $V$ the boundary of $V$, by $\operatorname{conv}(V)$ the convex hull of $V$, i.e. the set $\{x \in E: x=$ $\sum_{i=1}^{n} t_{i} v_{i}$ and $\left.v_{i} \in V, t_{i} \in[0,1], \sum_{i=1}^{n} t_{i}=1\right\}$, and $\overline{\operatorname{conv}}(V)=\operatorname{cl} \operatorname{conv}(V)$. Moreover, $B_{E}\left(x_{0}, r\right)=\left\{x \in E:\left\|x_{0}-x\right\|_{E} \leq r\right\}$.

1. $u$-Fundamentally contractible maps and morphisms. Let $E, E^{\prime}$ be Banach spaces, $X, V$ be subsets of $E$ such that $X \subset V$, and $Y$ be a closed convex subset of $E^{\prime}$. Moreover, let $u: V \rightarrow Y$ be a proper $\left({ }^{1}\right)$ continuous (singlevalued) map and $\varphi: X \multimap Y$ be a multivalued map.

Definition 1.1. A nonempty closed convex set $K \subset Y$ is called $u$-fundamental for $\varphi$ provided

(i) $\varphi\left(u^{-1}(K) \cap X\right) \subset K$,

(ii) if $u(x) \in \overline{\operatorname{conv}}(\varphi(x) \cup K)$, then $u(x) \in K$.

Observe that if $E=E^{\prime}$ and $u=\operatorname{id}_{E}$ is the identity on $E$, then $K$ is nothing else but a fundamental set for $\varphi$ in the sense of e.g. [3].

Properties of $u$-fundamental sets are summarized in the following result.

Theorem 1.2. (i) If $K$ is a u-fundamental set for $\varphi$, then $\{x \in X$ : $u(x) \in \varphi(x)\} \subset u^{-1}(K)$.

(ii) If $K_{1} \cap K_{2} \neq \emptyset$ and $K_{1}, K_{2}$ are $u$-fundamental sets for $\varphi$, then so is $K=K_{1} \cap K_{2}$.

(iii) If $P \subset K$ and $K$ is a u-fundamental set for $\varphi$, then so is $K^{\prime}=$ $\overline{\operatorname{conv}}\left(\varphi\left(u^{-1}(K) \cap X\right) \cup P\right)$.

$\left({ }^{1}\right)$ That is, $u^{-1}(C)$ is compact for any compact set $C \subset Y$. 
(iv) If $K$ is the intersection of all u-fundamental sets for $\varphi$, then $K=$ $\overline{\operatorname{conv}}\left(\varphi\left(u^{-1}(K) \cap X\right)\right)$.

(v) For any $A \subset Y$, there exists a u-fundamental set $K$ such that $K=$ $\overline{\operatorname{conv}}\left(\varphi\left(u^{-1}(K) \cap X\right) \cup A\right)$.

Proof. Only part (v) requires a proof. It is obvious that $Y$ is a $u$-fundamental set for $\varphi$. Put

$$
K=\bigcap_{L \in \mathcal{K}} L,
$$

where $\mathcal{K}=\{L \subset Y: L$ is a $u$-fundamental set for $\varphi$ and $A \subset L\}$. We see at once that $K$ is a $u$-fundamental set for $\varphi, A \subset K$ and

$$
K^{\prime}=\overline{\operatorname{conv}}\left(\varphi\left(u^{-1}(K) \cap X\right) \cup A\right) \subset K .
$$

But $K^{\prime} \in \mathcal{K}$, which follows from part (iii). By the definition of $K, K \subset K^{\prime}$, which completes the proof.

Observe that the set $K$ from (v) of the above theorem is the smallest $u$-fundamental set for $\varphi$ containing $A$.

DeFINITION 1.3. We say that $\varphi$ is a $u$-fundamentally contractible map if there exists a compact $u$-fundamental set for $\varphi$.

Remark 1.4. Let $E^{\prime \prime}$ be a Banach space. Assume that $Z: E^{\prime} \rightarrow E^{\prime \prime}$ is a continuous linear isomorphism. If $\varphi: X \multimap Y$ is $u$-fundamentally contractible, then $Z \circ \varphi$ is $(Z \circ u)$-fundamentally contractible. Indeed, if $K$ is a compact $u$-fundamental set for $\varphi$, then $Z(K)$ is a compact $Z \circ u$-fundamental set for $Z \circ \varphi$.

It is clear that without additional knowledge about the structure of a mapping and its values no satisfactory approximation or algebraic methods are available. Observe that any multivalued map $\varphi: X \multimap Y$ may be represented by the formula

$$
\varphi(x)=q_{\varphi}\left(p_{\varphi}^{-1}(x)\right)
$$

for $x \in X$, where

$$
X \stackrel{p_{\varphi}}{\longleftarrow} \operatorname{Gr}(\varphi) \stackrel{q_{\varphi}}{\longrightarrow} Y,
$$

$\operatorname{Gr}(\varphi)=\{(x, y) \in X \times Y: y \in \varphi(x)\}$ is the graph of $\varphi$ and $p_{\varphi}, q_{\varphi}$ are the projections. Note that, in view of the upper semicontinuity of $\varphi, p_{\varphi}$ is proper as a closed surjection with compact fibers.

Clearly $\varphi$ may admit other factorizations of the form $X \stackrel{p}{\leftarrow} W \stackrel{q}{\rightarrow} Y$ (i.e. $\left.\varphi(x)=q\left(p^{-1}(x)\right), x \in X\right)$ where $p, q$ are no longer projections, but $p$ is still a proper surjection.

As usual, we will need some assumptions concerning the values of the maps considered. In the described situation some additional assumptions on $p$ (more precisely, on its fibers) imply suitable properties of the values of $\varphi$. 
Therefore, from now on we will consider only those multivalued maps which can be factorized by means admissible cotriads (in the sense defined below).

Definition 1.5. Let $W$ be a space. We say that a cotriad $X \stackrel{p}{\leftarrow} W \stackrel{q}{\rightarrow} Y$, also denoted by $(p, q)$, is admissible if

(i) $q$ is a singlevalued map,

(ii) $p$ is a singlevalued proper map,

(iii) $p^{-1}(x)$ is a cell-like set for every $x \in X$.

Recall that a set $A \subset W$ is called cell-like if it is compact and if for any embedding $e: A \hookrightarrow Y$ into an absolute neighborhood retract $Y$, the set $e(A)$ is contractible in $Y$ (see [2] and [16]).

REMARK 1.6. Condition (iii) may be replaced by

$(\text { iii) })^{\prime} \sup _{x \in X} \operatorname{dim} p^{-1}(x)<\infty$ and for each $x \in X$, the fiber $p^{-1}(x)$ is acyclic with respect to Cech cohomology $\left({ }^{2}\right)$.

Of course, in this way we get a different class of multivalued maps, but it is also appropriate for all considerations that follow. In both classes, values of maps may be nonconvex.

Observe that each admissible cotriad $(p, q)$ generates a u.s.c. multivalued $\operatorname{map} \varphi_{(p, q)}: X \multimap Y, \varphi(x)=q\left(p^{-1}(x)\right)$, so we can define the following notions.

Definition 1.7. (i) A closed convex set $K$ is called $u$-fundamental for an admissible cotriad $(p, q)$ if it is $u$-fundamental for the multivalued map $\varphi_{(p, q)}$.

(ii) The cotriad $(p, q)$ is called $u$-fundamentally contractible if so is the $\operatorname{map} \varphi_{(p, q)}$.

Definition 1.8. We denote by $\mathcal{D}(X, Y, u)$ the class of all admissible cotriads

$$
X \stackrel{p}{\leftarrow} W \stackrel{q}{\rightarrow} Y
$$

such that

(i) $(p, q)$ is $u$-fundamentally contractible,

(ii) if $K, K^{\prime}$ are two compact disjoint $u$-fundamental sets for $(p, q)$, then there exist a finite number of compact $u$-fundamental sets $K_{1}, \ldots, K_{n}$ such that $K \cap K_{1} \neq \emptyset, K_{n} \cap K^{\prime} \neq \emptyset$ and $K_{i} \cap K_{i+1} \neq \emptyset$.

Observe that if we know a priori that $\left\{x \in X: u(x) \in q\left(p^{-1}(x)\right)\right\} \neq \emptyset$, then any two $u$-fundamental sets have nonempty intersection (see Theorem 1.2). However we need to impose condition (ii) in Definition 1.8 in

$\left(^{2}\right)$ That is, $\mathcal{H}^{*}\left(p^{-1}(x)\right)=\mathcal{H}^{*}(\mathrm{pt})$, where pt is a one-point space and $\mathcal{H}^{*}$ denotes Čech cohomology. 
order to have a relation between different $u$-fundamental sets. As shown by the examples given below, this condition does not restrict generality.

EXAMPLE 1.9 (Compact cotriads). If $(p, q)$ is compact (i.e. $\operatorname{cl} q\left(p^{-1}(X)\right)$ is compact), then $(p, q) \in \mathcal{D}(X, Y, u)$. Indeed, $K=\overline{\operatorname{conv}}\left(q\left(p^{-1}(X)\right)\right)$ is a compact $u$-fundamental set for $(p, q)$. If there exists a compact $u$-fundamental set $K^{\prime}$ such that $K \cap K^{\prime}=\emptyset$, then the set

$$
K^{\prime \prime}=\overline{\operatorname{conv}}\left(q\left(p^{-1}(X)\right) \cup\{v\}\right),
$$

where $v \in K^{\prime}$, is a compact $u$-fundamental set for $(p, q)$ (because in fact $\left.K^{\prime \prime}=\overline{\operatorname{conv}}\left(q\left(p^{-1}\left(u^{-1}(Y) \cap X\right)\right) \cup\{v\}\right)\right)$ and it has nonempty intersections with $K$ and $K^{\prime}$. We denote by $\mathcal{D}_{\mathrm{c}}(X, Y, u)$ the subclass of $\mathcal{D}(X, Y, u)$ consisting of the compact cotriads.

EXAMPLE 1.10 ( $u$-Condensing cotriads). Recall that a measure of noncompactness in a Banach space $E^{\prime}$ is a function $\mu: \mathcal{B} \rightarrow[0, \infty)$ defined on the family of all bounded subsets of $E^{\prime}$ and having the following properties:

(1) $\mu(A)=0$ if and only if $\operatorname{cl} A$ is compact.

(2) $\mu$ is a "seminorm", i.e. $\mu(\lambda \cdot A)=|\lambda| \mu(A)$ and $\mu\left(A_{1}+A_{2}\right) \leq \mu\left(A_{1}\right)+$ $\mu\left(A_{2}\right)$.

(3) If $A_{1} \subset A_{2}$, then $\mu\left(A_{1}\right) \leq \mu\left(A_{2}\right), \mu\left(A_{1} \cup A_{2}\right) \leq \max \left\{\mu\left(A_{1}\right), \mu\left(A_{2}\right)\right\}$.

(4) $\mu(\operatorname{conv} A)=\mu(A)$ and $\mu(\operatorname{cl} A)=\mu(A)$.

(5) If a sequence $\left\{A_{i}\right\}_{i=1}^{\infty}$ is decreasing and $\lim _{i \rightarrow \infty} \mu\left(A_{i}\right)=0$, then $A_{\infty}=\bigcap_{i=1}^{\infty} \operatorname{cl} A_{i}$ is a compact nonempty set.

Assume that both $u$ and the map determined by $(p, q)$ are bounded and that $(p, q)$ is $u$-condensing, i.e. for any $A \subset X$, if $\mu\left(q\left(p^{-1}(A)\right)\right) \geq \mu(u(A))$ then $\mathrm{cl} A$ is compact. Then $(p, q) \in \mathcal{D}(X, Y, u)$.

Indeed, let $y \in q\left(p^{-1}(X)\right)$. By Theorem 1.2(v), there exists a $u$-fundamental set $K$ such that $K=\overline{\operatorname{conv}}\left(q\left(p^{-1}\left(u^{-1}(K)\right) \cap X\right) \cup\{y\}\right)$. Observe that $K$ is bounded. Assume that $K$ is not compact. Then

$$
\begin{aligned}
\mu(K) & =\mu\left(\overline{\operatorname{conv}}\left(q\left(p^{-1}\left(u^{-1}(K) \cap X\right)\right) \cup\{y\}\right)\right) \\
& =\mu\left(q\left(p^{-1}\left(u^{-1}(K) \cap X\right)\right) \cup\{y\}\right) \\
& =\mu\left(q\left(p^{-1}\left(u^{-1}(K) \cap X\right)\right)\right)<\mu\left(u\left(u^{-1}(K) \cap X\right)\right) \leq \mu(K),
\end{aligned}
$$

a contradiction.

Now, for any two compact $u$-fundamental sets $K_{1}, K_{2}$ and $x \in K_{1}$, $y \in K_{2}$, there exists a $u$-fundamental set $K_{3}$ such that

$$
K_{3}=\overline{\operatorname{conv}}\left(\left(q\left(p^{-1}\left(K_{3}\right) \cap X\right)\right) \cup\{x, y\}\right) .
$$

As above we prove that $K_{3}$ is compact.

EXAMPLE 1.11 ( $u$-Set contractions). A cotriad $(p, q)$ is called a $u$-set contraction if there exists $k \in(0,1)$ such that for any bounded $A \subset X$, 
$\mu\left(q\left(p^{-1}(A)\right)\right) \leq k \mu(u(A))$. Then, clearly, $(p, q)$ is $u$-condensing and hence $(p, q) \in \mathcal{D}(X, Y, u)$.

EXAMPLE 1.12 ( $u$-Limit compact cotriads). Let $Y=u(X)$ and $K_{1}=$ $\overline{\operatorname{conv}}\left(q\left(p^{-1}(X)\right)\right), K_{i}=\overline{\operatorname{conv}}\left(q\left(p^{-1}\left(u^{-1}\left(K_{i-1}\right) \cap X\right)\right)\right)$ for $i>1$. The cotriad $(p, q)$ is $u$-limit compact if the set

$$
K=\bigcap_{i=1}^{\infty} K_{i}
$$

is nonempty and compact. Obviously, $K$ is a $u$-fundamental set for $(p, q)$. Assume that $L_{0} \subset Y$ is another compact $u$-fundamental set for $(p, q)$. Of course $u^{-1}\left(L_{0}\right) \cap X \neq \emptyset$. For $i=1,2, \ldots$, put

$$
L_{i}=\overline{\operatorname{conv}}\left(q\left(p^{-1}\left(u^{-1}\left(L_{i-1}\right) \cap X\right)\right)\right), \quad i \in \mathbb{N} \backslash\{0\},
$$

and observe that

$$
\emptyset \neq L_{1} \subset \overline{\operatorname{conv}}\left(q\left(p^{-1}(X)\right)\right)=K_{1}
$$

and, for $i>1$,

$$
\emptyset \neq L_{i} \subset \overline{\operatorname{conv}}\left(q\left(p^{-1}\left(u^{-1}\left(K_{i-1}\right) \cap X\right)\right)\right)=K_{i} \subset u(X) .
$$

Moreover $L_{i} \subset L_{0} \cap K_{i}$, hence $L_{0} \cap K \neq \emptyset$.

EXAMPLE 1.13. If $u=\mathrm{id}_{X}$ and $E=E^{\prime}$, then every $u$-condensing cotriad is condensing, every $u$-set contraction is a $k$-set contraction and every $u$-limit compact map is limit compact.

EXAMPLE 1.14. First we generalize the definition of $\mathcal{K}_{n}$-operators (cf. [1]). Let $\mathcal{A} \subset 2^{E^{\prime}}$. A multivalued map $\varphi: X \multimap Y$ is called an $(u, \mathcal{A})$-operator if for any $T \in \mathcal{A}$ and $Z \subset Y$, the following condition holds:

if $\quad \overline{\operatorname{conv}}\left(\varphi\left(u^{-1}(Z) \cap X\right) \cup T\right)=Z, \quad$ then $\quad Z$ is compact.

Let $\mathcal{K}_{n}$ denote the family of all $n$-element subsets of $Y$, and $\mathcal{K}_{\infty}, \mathcal{K}_{c}$ the families of all finite and all compact subsets of $Y$, respectively.

If $\mathcal{A}$ is an arbitrary family among $\mathcal{K}_{2}, \ldots, \mathcal{K}_{n}, \ldots, \mathcal{K}_{\infty}, \mathcal{K}_{\mathrm{c}}$, and $\varphi_{(p, q)}$ is a $(u, \mathcal{A})$-operator, then $(p, q) \in \mathcal{D}(X, Y, u)$. The proof is easy if one recalls properties of $u$-fundamental sets (Theorem 1.2).

As mentioned earlier, a multivalued map can be factorized by different cotriads. To reduce this freedom we define the following equivalence relation in $\mathcal{D}(X, Y, u)$.

DeFINITION 1.15. We say that cotriads

$$
X \stackrel{p_{i}}{\longleftarrow} W_{i} \stackrel{q_{i}}{\longrightarrow} Y, \quad i=1,2,
$$


from $\mathcal{D}(X, Y, u)$ are equivalent (written $\left(p_{1}, q_{1}\right) \approx\left(p_{2}, q_{2}\right)$ ) if there exists a homeomorphism $f: W_{1} \rightarrow W_{2}$ such that the following diagram is commutative:

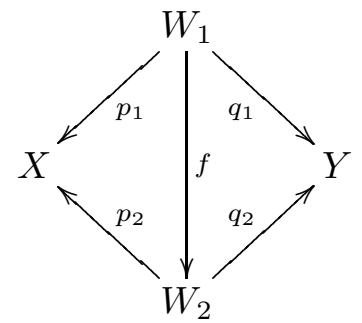

Define

$$
\mathcal{M}(X, Y, u)=\mathcal{D}(X, Y, u) / \approx .
$$

Elements of $\mathcal{M}(X, Y, u)$ will be called $u$-fundamentally contractible morphisms and will be denoted by Greek capital letters $\Phi, \Psi, \ldots$

Any morphism $\Phi \in \mathcal{M}(X, Y, u)$ determines a multivalued map $\varphi_{\Phi}$ : $X \multimap E^{\prime}$ by $\varphi_{\Phi}(x)=q\left(p^{-1}(x)\right)$, where $(p, q) \in \Phi$. The definition does not depend on the choice of $(p, q)$, so $\varphi_{\Phi}$ is well defined. From now on we write $\Phi(x)$ for $\varphi_{\Phi}(x)$.

REMARK 1.16. Assume that $\left(p_{1}, q_{1}\right) \approx\left(p_{2}, q_{2}\right)$. Then

(i) $\left\{x \in X: u(x) \in q_{1}\left(p_{1}^{-1}(x)\right)\right\}=\left\{x \in X: u(x) \in q_{2}\left(p_{2}^{-1}(x)\right)\right\}$,

(ii) if $K$ is a $u$-fundamental set for $\left(p_{1}, q_{1}\right)$, then it is a $u$-fundamental set for $\left(p_{2}, q_{2}\right)$, so we can call $K$ a $u$-fundamental set for the morphism $\Phi=\left[\left(p_{1}, q_{1}\right)\right]$ and, of course, $K$ is a $u$-fundamental set for $\varphi_{\Phi}$.

If $f: X \rightarrow Y$ is a $u$-fundamentally contractible singlevalued map, then we can identify $f$ with a morphism $\left[\left(\operatorname{id}_{X}, f\right)\right]$ from $\mathcal{M}(X, Y, u)$. Let $\Phi$ be represented by the cotriad $Y \stackrel{p}{\leftarrow} W \stackrel{q}{\rightarrow} Z$. The composition $\Phi \circ f$ is the morphism represented by the cotriad

$$
X \stackrel{\widetilde{p}}{\leftarrow} X \otimes W \stackrel{q \circ \widetilde{f}}{\longrightarrow} Z,
$$

where $X \otimes W=\{(x, w) \in X \times W: p(w)=f(x)\}, \widetilde{p}(x, w)=x$ and $\widetilde{f}(x, w)=w$.

Let $I=[0,1], i_{j}: X \rightarrow X \times I, j=0,1$, be the embeddings given by $i_{j}(x)=(x, j), x \in X$, and $v: X \times I \rightarrow Y$ be defined by $v(x, t)=u(x)$. For any fixed $t \in I$ we may identify $v(\cdot, t)$ and $u$.

Definition 1.17. Let $K$ be a compact convex subset of $Y$. Morphisms $\Phi_{0}, \Phi_{1} \in \mathcal{M}(X, Y, u)$ are $(u, K)$-homotopic (written $\Phi_{0} \simeq_{K} \Phi_{1}$ ) if there exists a morphism $\Phi \in \mathcal{M}(X \times I, Y, v)$ such that for every $t \in I$ and $(P, Q) \in$ $\Phi, K$ is a $u$-fundamental set for $Q\left(P^{-1}(\cdot, t)\right)$ and $\Phi \circ i_{j}=\Phi_{j}, j=0,1$. 
Definition 1.18. Morphisms $\Phi_{0}, \Phi_{1} \in \mathcal{M}(X, Y, u)$ are $u$-homotopic (written $\Phi_{0} \simeq \Phi_{1}$ ) if there exist a finite number of compact convex sets $K_{1}, \ldots, K_{n} \subset Y$ and morphisms $\Psi_{1}, \ldots, \Psi_{n-1}$ such that

$$
\Phi_{0} \simeq_{K_{1}} \Psi_{1} \simeq_{K_{2}} \ldots \simeq_{K_{n-1}} \Psi_{n-1} \simeq_{K_{n}} \Phi_{1} .
$$

We end this section with the notion of compact $u$-homotopy. Let $\mathcal{M}_{c}(X, Y, u) \subset \mathcal{M}(X, Y, u)$ be the set of compact morphisms, i.e. such that $\mathcal{M}_{c}(X, Y, u)=\mathcal{D}_{c}(X, Y, u) / \approx($ see 1.9$)$.

Definition 1.19. Morphisms $\Phi_{0}, \Phi_{1} \in \mathcal{M}_{\mathrm{c}}(X, Y, u)$ are compactly $u$-homotopic if there exists a morphism $\Phi \in \mathcal{M}_{\mathrm{c}}(X \times I, Y, v)$ such that $\Phi \circ i_{j}=\Phi_{j}$

Of course, if morphisms are compactly $u$-homotopic, then they are $(u, K)$-homotopic with $K=\overline{\operatorname{conv}}\left(\varphi_{\Phi}(X \times I)\right)$, hence simply $u$-homotopic.

2. Associated compact cotriads. Let $E, E^{\prime}, X, Y, u$ be as in the previous section. Take $\Phi \in \mathcal{M}(X, Y, u)$ and a cotriad $X \stackrel{p}{\leftarrow} W \stackrel{q}{\rightarrow} Y$ such that $(p, q) \in \Phi$.

Definition 2.1. Let $K$ be a compact $u$-fundamental set for $(p, q)$. A cotriad $X \stackrel{p}{\leftarrow} W \stackrel{\bar{q}}{\rightarrow} K$ such that $\bar{q}$ is a compact map and $\bar{q}_{\mid p^{-1}\left(u^{-1}(K) \cap X\right)}=$ $q_{\mid p^{-1}\left(u^{-1}(K) \cap X\right)}$ is said to be associated with $(p, q)$ with respect to $K$.

Obviously, the set $K$ is $u$-fundamental for $(p, \bar{q})$, and $(p, \bar{q}) \in \mathcal{D}_{\mathrm{c}}(X, Y, u)$.

It is easily seen that for any $(p, q) \in \Phi \in \mathcal{M}(X, Y, u)$ there exists a compact associated cotriad. Indeed, if $K$ is a $u$-fundamental set for $(p, q)$, then the map

$$
q_{\mid p^{-1}\left(u^{-1}(K) \cap X\right)}: p^{-1}\left(u^{-1}(K) \cap X\right) \rightarrow K
$$

admits a compact extension $q^{\prime}: W \rightarrow K$ and $\left(p, q^{\prime}\right)$ satisfies the above definition.

Let us collect some properties of associated cotriads.

Lemma 2.2. (i) Let $(p, q) \in \mathcal{D}(X, Y, u), K \subset Y$ be a compact u-fundamental set for $(p, q)$, and $(p, \bar{q})$ be a compact cotriad associated with $(p, q)$ with respect to $K$. Then $\left\{x \in X: u(x) \in q\left(p^{-1}(x)\right)\right\}=\{x \in X: u(x) \in$ $\left.\bar{q}\left(p^{-1}(x)\right)\right\}$.

(ii) Let $K_{1}, K_{2}$ be compact $u$-fundamental sets for $(p, q)$ and $K_{1} \subset K_{2}$. If $(p, \bar{q})$ is a cotriad associated with $(p, q)$ with respect to $K_{2}$, then $K_{1}$ is a $u$-fundamental set for $(p, \bar{q})$.

(iii) Morphisms $[(p, q)]$ and $[(p, \bar{q})]$ are u-homotopic. If , additionally, $\left\{x \in X: u(x) \in q\left(p^{-1}(x)\right)\right\} \cap \mathrm{bd} X=\emptyset$, then the multivalued map determined by the u-homotopy joining these cotriads also has no coincidence points with $u$ on $\mathrm{bd} X$. 
Pro of. Parts (i) and (ii) are obvious.

To prove (iii), let $r: E^{\prime} \rightarrow K$ be a retraction. Consider the cotriad $X \times I \stackrel{P}{\leftarrow} W \times I \stackrel{Q}{\rightarrow} Y$ given by

$$
\begin{aligned}
& P(w, t)=(p(w), t), \\
& Q(w, t)= \begin{cases}(1-2 t) q(w)+2 t(r \circ q)(w) & \text { for } t \in[0,1 / 2], \\
(2-2 t)(r \circ q)(w)+(2 t-1) \bar{q}(w) & \text { for } t \in(1 / 2,1]\end{cases}
\end{aligned}
$$

An easy computation shows that $[(P, Q)] \circ i_{0}=[(p, q)],[(P, Q)] \circ i_{1}=$ $[(p, \bar{q})]$ and for every $t \in I, K$ is a $u$-fundamental set for $Q\left(P^{-1}(\cdot, t)\right)$ and $\left\{x \in X: u(x) \in Q\left(P^{-1}(x, t)\right)\right\} \cap \operatorname{bd} X=\emptyset$.

Moreover, observe that if $(p, q)$ and $u$ have no coincidence points on $X$, then also $(P, Q)$ has no coincidence points with $v(x, t):=u(x)$ on $X \times I$.

From the above lemma it follows, in particular, that if

$$
\left\{x \in X: u(x) \in q\left(p^{-1}(x)\right)\right\} \cap \operatorname{bd} X=\emptyset,
$$

then also

$$
\left\{x \in X: u(x) \in \bar{q}\left(p^{-1}(x)\right)\right\} \cap \operatorname{bd} X=\emptyset .
$$

Let $\Phi \in \mathcal{M}(X, Y, u)$ and $K$ be a $u$-fundamental set for $\Phi$ (see Remark 1.16). One can define a compact morphism $\bar{\Phi}$ associated with $\Phi$ with respect to $K$ by putting $\bar{\Phi}=[(p, \bar{q})]$, where $(p, \bar{q})$ is a compact cotriad associated with $(p, q) \in \Phi$ with respect to $K$. The following theorems show that any two compact morphisms associated with $\Phi$ are compactly $u$-homotopic.

TheOREM 2.3. Let $\left(p, \bar{q}_{0}\right),\left(p, \bar{q}_{1}\right)$ be compact cotriads associated with $(p, q) \in \mathcal{D}(X, Y, u)$ with respect to compact $u$-fundamental sets $K_{0}, K_{1}$, respectively. Assume that $(p, q)$ and $u$ have no coincidence points on $\operatorname{bd} X$. Then the morphisms $\left[\left(p, \bar{q}_{0}\right)\right],\left[\left(p, \bar{q}_{1}\right)\right]$ are compactly u-homotopic and the map determined by the homotopy has no coincidence points with $u$ on $\operatorname{bd} X$.

Proof. Without loss of generality assume that $K_{2}=K_{0} \cap K_{1} \neq \emptyset$ (cf. Definition 1.8). Of course, $K_{2}$ is a compact $u$-fundamental set for $(p, q)$. Let $r_{i}: E^{\prime} \rightarrow K_{i}, i=0,1,2$, be retractions. Consider the cotriad $X \times I \stackrel{P}{\leftarrow}$ $W \times I \stackrel{Q}{\rightarrow} K_{0} \cup K_{1}$ defined by

$$
\begin{aligned}
& P(w, t)=(p(w), t), \\
& Q(w, t)= \begin{cases}(1-4 t) \bar{q}_{0}(w)+4 t\left(r_{0} \circ q\right)(w) & \text { for } t \in[0,1 / 4], \\
(2-4 t)\left(r_{0} \circ q\right)(w)+(4 t-1)\left(r_{2} \circ q\right)(w) & \text { for } t \in(1 / 4,1 / 2], \\
(3-4 t)\left(r_{2} \circ q\right)(w)+(4 t-2)\left(r_{1} \circ q\right)(w) & \text { for } t \in(1 / 2,3 / 4], \\
(4-4 t)\left(r_{1} \circ q\right)(w)+(4 t-3) \bar{q}_{1}(w) & \text { for } t \in(3 / 4,1] .\end{cases}
\end{aligned}
$$

Obviously, $(P, Q) \in \mathcal{D}_{\mathrm{c}}(X \times I, Y, v)$, where, as above, $v: X \times I \rightarrow Y$ is given by $v(x, t)=u(x)$. We prove that, for any $t \in I,\{x \in \operatorname{bd} X: u(x) \in$ 
$\left.Q\left(P^{-1}(x, t)\right)\right\}=\emptyset$. Assume that $u(x) \in Q\left(P^{-1}(x, t)\right)=Q(\{(w, s) \in W \times I$ : $p(w)=x, s=t\})$.

If $t \in[0,1 / 4]$, then there exists $w \in W$ such that

$$
u(x)=(1-4 t) \bar{q}_{0}(w)+4 t\left(r_{0} \circ q\right)(w) \text { and } p(w)=x .
$$

By the above, $u(x) \in K_{0}$ and hence $x \in u^{-1}\left(K_{0}\right) \cap X$. Since $p(w)=x$, we have $w \in p^{-1}\left(u^{-1}\left(K_{0}\right) \cap X\right)$. This gives $\bar{q}_{0}(w)=q(w)$, and $q(w)=r_{0} \circ q(w)$, because $q(w) \in K_{0}$. Therefore, $Q(w, t)=q(w)$ and, consequently, $x \notin \mathrm{bd} X$.

The proof for $t \in(3 / 4,1]$ is similar.

If $t \in(1 / 4,1 / 2]$, then there exists $w \in W$ such that

$$
u(x)=(2-4 t)\left(r_{0} \circ q\right)(w)+(4 t-1)\left(r_{2} \circ q\right)(w) \quad \text { and } \quad p(w)=x .
$$

As above, $u(x) \in K_{0}$, so $w \in p^{-1}\left(u^{-1}\left(K_{0}\right) \cap X\right)$. Hence $q(w) \in K_{0}$. This gives $r_{0} \circ q(w)=q(w) \in q\left(p^{-1}(x)\right)$. From this it follows that $u(x) \in$ $\overline{\operatorname{conv}}\left(q\left(p^{-1}(x)\right) \cup K_{2}\right)$, because $r_{2} \circ q(w) \in K_{2}$. Since $K_{2}$ is a $u$-fundamental set for $(p, q), u(x) \in K_{2}$. It follows that $x \in u^{-1}\left(K_{2}\right)$ and hence $w \in$ $p^{-1}\left(u^{-1}\left(K_{2}\right) \cap X\right)$, which implies that $q(w) \in K_{2}$, and finally, that $r_{2} \circ q(w)=$ $q(w)$. Therefore $u(x) \in q\left(p^{-1}(x)\right)$, and $x \notin \operatorname{bd} X$.

The same proof works for $t \in(1 / 2,3 / 4]$.

It is easy to verify that the morphism $[(P, Q)]$ is a $u$-homotopy joining $\left[\left(p, \bar{q}_{0}\right)\right]$ to $\left[\left(p, \bar{q}_{1}\right)\right]$ with $K_{2}$ being a $u$-fundamental set for all maps $Q\left(P^{-1}(\cdot, t)\right), t \in I$.

Theorem 2.4. Let $X \stackrel{p_{0}}{\longleftarrow} W_{0} \stackrel{q_{0}}{\longrightarrow} E^{\prime}, X \stackrel{p_{1}}{\longleftarrow} W_{1} \stackrel{q_{1}}{\longrightarrow} E^{\prime},\left(p_{0}, q_{0}\right),\left(p_{1}, q_{1}\right)$ $\in \mathcal{D}(X, Y, u)$ and $\left(p_{0}, \bar{q}_{0}\right),\left(p_{1}, \bar{q}_{1}\right)$ be cotriads associated with $\left(p_{0}, q_{0}\right)$ and $\left(p_{1}, q_{1}\right)$, respectively. If $\left(p_{0}, q_{0}\right),\left(p_{1}, q_{1}\right)$ are equivalent, then the morphisms $\left[\left(p_{0}, \bar{q}_{0}\right)\right],\left[\left(p_{1}, \bar{q}_{1}\right)\right]$ are compactly u-homotopic.

Proof. Since $\left(p_{0}, q_{0}\right)$ and $\left(p_{1}, q_{1}\right)$ are equivalent, there exists a homeomorphism $f: W_{0} \rightarrow W_{1}$ such that $p_{1} \circ f=p_{0}$ and $q_{1} \circ f=q_{0}$ (see Definition $1.15)$ and we can assume that $\left(p_{0}, \bar{q}_{0}\right),\left(p_{1}, \bar{q}_{1}\right)$ are associated with $\left(p_{0}, q_{0}\right)$ and $\left(p_{1}, q_{1}\right)$ with respect to the same $u$-fundamental set $K$ (cf. Remark 1.16). Observe that $\left(p_{1}, \bar{q}_{1}\right),\left(p_{0}, \bar{q}_{1} \circ f\right)$ are equivalent in $\mathcal{D}_{\mathrm{c}}(X, Y, u)$. The morphism $[(P, Q)] \in \mathcal{M}_{\mathrm{c}}(X \times I, Y, v)$, where $v: X \times I \rightarrow Y, v(x, t)=u(x)$ and

$$
X \times I \stackrel{P}{\leftarrow} W_{0} \times I \stackrel{Q}{\rightarrow} K_{0},
$$

defined by

$$
P(w, t)=\left(p_{0}(w), t\right), \quad Q(w, t)=(1-t)\left(\bar{q}_{1} \circ f\right)(w)+t \bar{q}_{0}(w),
$$

gives a compact $u$-homotopy between $\left[\left(p_{0}, \bar{q}_{1} \circ f\right)\right]$ and $\left[\left(p_{0}, \bar{q}_{0}\right)\right]$. But we have $\left[\left(p_{0}, \bar{q}_{1} \circ f\right)\right]=\left[\left(p_{1}, q_{1}\right)\right]$ and the proof is complete. 
REMARK 2.5. If $\left(p_{0}, q_{0}\right)$ and $\left(p_{1}, q_{1}\right)$ are as in the above theorem, then $\left\{x \in X: u(x) \in q_{0}\left(p_{0}^{-1}(x)\right)\right\} \cap \operatorname{bd} X=\emptyset$ if and only if $\{x \in X: u(x) \in$ $\left.q_{1}\left(p_{1}^{-1}(x)\right)\right\} \cap \operatorname{bd} X=\emptyset$. Moreover, the homotopy defined in the proof of Theorem 2.4 and $v$ have no coincidence point on $\operatorname{bd} X$.

3. The coincidence index. Let $E, E^{\prime}$ be Banach spaces and $F: E \rightarrow E^{\prime}$ be a Fredholm linear operator of nonnegative index $k=\operatorname{ind}(F)$.

Recall that a bounded linear operator $F: E \rightarrow E^{\prime}$ is called a Fredholm operator if $\operatorname{dim} \operatorname{Ker}(F)<\infty$ and $\operatorname{dim} \operatorname{Coker}(F)<\infty$. Here, $\operatorname{Ker}(F)=$ $\{x \in E: F(x)=0\}$ is the null space of $F$ and $\operatorname{Coker}(F)=E^{\prime} / R(F)$ where $R(F):=F(E)$ is the range of $F$. Note that $R(F)$ is a closed subspace of $E^{\prime}$ (see IV.2.6 of [7]). The index of a Fredholm operator $F$ is the number

$$
\operatorname{ind}(F):=\operatorname{dim} \operatorname{Ker}(F)-\operatorname{dim} \operatorname{Coker}(F) .
$$

Definition 3.1. We denote by $\mathcal{M}^{F}\left(E, E^{\prime}\right)$ the collection of pairs $(\Phi, U)$ such that $U$ is an open bounded subset of $E, \Phi \in \mathcal{M}\left(\operatorname{cl} U, E^{\prime},\left.F\right|_{\mathrm{cl} U}\right)$ and $F(x) \notin \Phi(x)$ for all $x \in \operatorname{bd} U$.

To simplify the notation, from now on, if $(\Phi, U) \in \mathcal{M}^{F}\left(E, E^{\prime}\right)$, then an $\left.F\right|_{\mathrm{cl} U}$-fundamental set for $\Phi$ (resp. for $(p, q) \in \Phi$ or for $\left.\varphi_{\Phi}\right)$ will be called an $F$-fundamental set for $\Phi$ (resp. for $(p, q) \in \Phi$ or for $\varphi_{\Phi}$ ).

We say that the pairs $\left(\Phi_{0}, U\right),\left(\Phi_{1}, U\right)$ are $F$-homotopic (resp. compactly $F$-homotopic) if they are $u$-homotopic (resp. compactly $u$-homotopic) with $u=\left.F\right|_{\mathrm{cl} U}$ (see 1.18 and 1.19), and the $u$-homotopy has no coincidence points with $F$ on the boundary of $U$.

In this section we construct a general coincidence index, which is a homotopy invariant count of coincidence points of $F$ and multivalued maps determined by a morphism from $\mathcal{M}^{F}\left(E, E^{\prime}\right)$.

Definition 3.2. By a coincidence index we understand a function $\operatorname{ind}_{F}$ which assigns to any pair $(\Phi, U)$ ( $\Phi$ is a single- or multivalued map or morphism) an element of the $k$ th stable homotopy group of spheres $\Pi_{k}$ and has the following properties:

(i) (Existence) If $\operatorname{ind}_{F}(\Phi, U) \neq 0$, then there is $x_{0} \in U$ such that $F\left(x_{0}\right) \in \Phi\left(x_{0}\right)$.

(ii) (Localization) If $U^{\prime} \subset U$ is open and $F(x) \notin \Phi(x)$ for $x \in \operatorname{cl} U \backslash U^{\prime}$, then $\operatorname{ind}_{F}(\Phi, U)=\operatorname{ind}_{F}\left(\left.\Phi\right|_{\mathrm{cl} U^{\prime}}, U^{\prime}\right)$.

(iii) (Additivity) If $U_{1}, U_{2}$ are open disjoint subsets of $U$ and $F(x) \notin \Phi(x)$ for $x \in \operatorname{cl} U \backslash\left(U_{1} \cup U_{2}\right)$, then $\operatorname{ind}_{F}(\Phi, U)=\operatorname{ind}_{F}\left(\left.\Phi\right|_{\operatorname{cl} U_{1}}, U_{1}\right)+\operatorname{ind}_{F}\left(\left.\Phi\right|_{\operatorname{cl} U_{2}}, U_{2}\right)$.

(iv) (Homotopy) If $\left(\Phi_{1}, U\right)$ is $F$-homotopic to $(\Phi, U)$, then $\operatorname{ind}_{F}(\Phi, U)=$ $\operatorname{ind}_{F}\left(\Phi_{1}, U\right)$.

(v) (Restriction) Let $G: E^{\prime} \rightarrow E^{\prime}$ be a bounded linear projection with $\operatorname{Ker}(G)=R(F)$. Suppose that $\Phi(\operatorname{cl} U) \subset T$, where $T$ is a closed subspace 
of $E^{\prime}$. $\operatorname{Then}_{\operatorname{ind}}(\Phi, U)=\operatorname{ind}_{F^{\prime}}\left(\Phi^{\prime}, U \cap T^{\prime}\right)$, where $T^{\prime}=F^{-1}(T+R(G))$, $\Phi^{\prime}=\left.\Phi\right|_{\mathrm{cl} U \cap T^{\prime}}$ and $F^{\prime}=\left.F\right|_{T^{\prime}}$.

In order to provide a construction of an index on $\mathcal{M}^{F}\left(E, E^{\prime}\right)$, we first recall it briefly in the case of compact maps (following [12]). Similar homotopy invariants (in a less general situation) were studied earlier in [17], [6], [4], [10], [9].

First, let $E=\mathbb{R}^{m}, E^{\prime}=\mathbb{R}^{n}$, where $n \leq m \leq 2 n-2$, and let $f: \operatorname{cl} U \rightarrow \mathbb{R}^{n}$ be a continuous (singlevalued) map such that $\{x \in \operatorname{bd} U: F(x)=f(x)\}=\emptyset$. We identify the unit sphere $S^{n}$ with the one-point compactification of $\mathbb{R}^{n}$, where $0 \in \mathbb{R}^{n}$ is identified with the south pole $s_{-1}$ of $S^{n}$. Since $S^{n} \backslash\left\{s_{-1}\right\}$ is an absolute retract, there exists a continuous map $g: S^{m} \backslash U \rightarrow S^{n} \backslash\left\{s_{-1}\right\}$ such that $\left.g\right|_{\text {bd } U}=\left.(F-f)\right|_{\text {bd } U}$. Define $g^{\prime}: S^{m} \rightarrow S^{n}$ by the formula

$$
g^{\prime}(x)= \begin{cases}g(x) & \text { for } x \in S^{m} \backslash U \\ F(x)-f(x) & \text { for } x \in U\end{cases}
$$

Take the homotopy class $\left[g^{\prime}\right]$ of $g^{\prime}$ in the $m$ th homotopy group of $S^{n}$, denoted by $\pi_{m}\left(S^{n}\right)$.

Let $k=m-n$. If $k+2 \leq n$, then we have the suspension isomorphism $\pi_{n+k}\left(S^{n}\right) \cong \pi_{n+k+1}\left(S^{n+1}\right)$, hence the $k$ th stable homotopy group of spheres $\Pi_{k}$ defined by

$$
\Pi_{k}=\lim _{n \geq 0} \pi_{n+k}\left(S^{n}\right)
$$

is isomorphic to $\pi_{n+k}\left(S^{n}\right)$ for sufficiently large $n$. The index $\operatorname{ind}_{F}(f, U)$ is the element of $\Pi_{k}$ corresponding to $\left[g^{\prime}\right]$ by an appropriate isomorphism.

The above procedure was suggested by [6] and introduced in [9]. It may be formulated in the language of cohomotopy groups (see [12]).

This index has all the standard properties (i)-(v) from 3.2. The proofs can be found in [12] and [6].

Now, let $E$ be an arbitrary Banach space, $E^{\prime}$ be a Banach space with a given orientation $\left({ }^{3}\right)$ and let $f: \operatorname{cl} U \rightarrow E^{\prime}$ be a compact map with $f(x) \neq F(x)$ for $x \in \operatorname{bd} U$. Using much more complex arguments, based on Gȩba's infinite-dimensional cohomotopy theory (see [5]) with suitable modifications, Kryszewski [12] has defined an index

$$
\operatorname{ind}_{F}(f, U) \in \Pi_{k},
$$

which has all properties from 3.2 and additionally the following one:

Property 3.3 (Boundary dependence). If $f, g: \operatorname{cl} U \rightarrow E^{\prime}$ and $\left.f\right|_{\mathrm{bd} U}=$ $\left.g\right|_{\text {bd } U}$, then $\operatorname{ind}_{F}(f, U)=\operatorname{ind}_{F}(g, U)$.

$\left({ }^{3}\right)$ An orientation in an infinite-dimensional Banach space $E^{\prime}$ is a family $\mathcal{O}=$ $\left\{\mathcal{O}_{L}\right\}_{L \in \Lambda}$, where $\Lambda$ is the family of all finite-dimensional linear subspaces of $E^{\prime}$ and $\mathcal{O}_{L}$ is a fixed orientation on $L \in \Lambda$ (cf. [5]). 
REMARK 3.4. If $E=E^{\prime}$ and $F=\mathrm{id}_{E}$, then $\operatorname{ind}_{\mathrm{id}_{E}}(g, U) \in \Pi_{0}=\mathbb{Z}$ is the Leray-Schauder fixed point index of $g$ on $U$.

Now we can describe the multivalued situation starting with the compact case (cf. [12], [14], [15]). We obtain the following theorem, which is a corollary of Theorem 4.51 and Remark 4.52 of [12].

Theorem 3.5. Let $\Phi \in \mathcal{M}_{\mathrm{c}}\left(\mathrm{cl} U, E^{\prime},\left.F\right|_{\mathrm{cl} U}\right)$ be such that $\{x \in \operatorname{bd} U$ : $F(x) \in \Phi(x)\}=\emptyset$ and let $i: \operatorname{bd} U \rightarrow \operatorname{cl} U$ be the inclusion. Then there exists

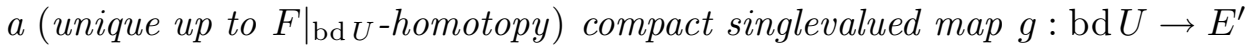
such that the morphism $\left[\left(\mathrm{id}_{\mathrm{bd} U}, g\right)\right]$ is $\left.F\right|_{\mathrm{bd} U}$-compactly homotopic to the morphism $\Phi \circ i$ and the map determined by the homotopy has no coincidence points with $F$.

The above theorem and Property 3.3 allow us to define a generalized coincidence index for compact morphisms.

Definition 3.6. Assume that $\Phi$ satisfies the assumptions of Theorem 3.5. The generalized coincidence index of $\Phi$ on $U$ with respect to $F$ is defined by

$$
\operatorname{ind}_{F}(\Phi, U):=\operatorname{ind}_{F}(\bar{g}, U)
$$

where $\bar{g}: \operatorname{cl} U \rightarrow E^{\prime}$ is an arbitrary compact extension of $g$.

Theorem 3.7 (see [12]). The index defined above has properties (i)-(v) of 3.2 .

Now, we are in a position to define a generalized coincidence index for morphisms from $\mathcal{M}^{F}\left(E, E^{\prime}\right)$.

Definition 3.8. Assume that $(\Phi, U) \in \mathcal{M}^{F}\left(E, E^{\prime}\right)$. The coincidence index of $\Phi$ on $U$ with respect to $F$ is

$$
\operatorname{ind}_{F}(\Phi, U):=\operatorname{ind}_{F}(\bar{\Phi}, U)
$$

where $\bar{\Phi}=[(p, \bar{q})]$ and $(p, \bar{q})$ is a compact cotriad associated with $(p, q) \in \Phi$.

The correctness of this definition follows from Theorems 2.3 and 2.4 (the index does not depend on the choice of $(p, q)$ and its associated cotriad). Obviously, $[(p, \bar{q})] \in \mathcal{M}_{\mathrm{c}}\left(\mathrm{cl} U, E^{\prime},\left.F\right|_{\mathrm{cl} U}\right)$.

Observe that if $(\Phi, U) \in \mathcal{M}^{F}\left(E, E^{\prime}\right)$ and $U^{\prime}$ is an open subset of $E$ such that $\operatorname{cl} U^{\prime} \subset U$, then also $\left(\left.\Phi\right|_{\mathrm{cl} U^{\prime}}, U^{\prime}\right) \in \mathcal{M}^{F}\left(E, E^{\prime}\right)$. Indeed, any $F$ fundamental set for $\Phi$ is an $F$-fundamental set for $\left.\Phi\right|_{U^{\prime}}$. This allows us to state the following theorem.

Theorem 3.9. Let $(\Phi, U) \in \mathcal{M}^{F}\left(E, E^{\prime}\right), \varphi_{\Phi}$ be the multivalued map determined by $\Phi$ and assume that $\varphi_{\Phi}$ has no coincidence points with $F$ on the boundary of $U$. Then the index $\operatorname{ind}_{F}$ from Definition 3.8 has all the properties $3.2(\mathrm{i})-(\mathrm{v})$. 
Pr o o f. As above, we denote by $\bar{\Phi}$ the morphism containing the compact cotriad associated with $(p, q) \in \Phi$.

(i) If $\operatorname{ind}_{F}(\Phi, U) \neq 0$, then $\operatorname{ind}_{F}(\bar{\Phi}, U) \neq 0$. Hence there exists $x_{0} \in U$ such that $F\left(x_{0}\right) \in \bar{\Phi}\left(x_{0}\right)$. But the sets of coincidence points are identical for $\Phi$ and $\bar{\Phi}$, so $F\left(x_{0}\right) \in \Phi\left(x_{0}\right)$.

(ii) Observe that if $(p, \bar{q})$ is a compact cotriad associated with $(p, q) \in \Phi$, then $\left.(p, \bar{q})\right|_{\mathrm{cl} U^{\prime}}$ is a compact cotriad associated with $\left.(p, q)\right|_{\mathrm{cl} U^{\prime}}$, and $\left.(p, \bar{q})\right|_{\mathrm{cl} U^{\prime}}$ $\left.\in \bar{\Phi}\right|_{\mathrm{cl} U^{\prime}}$. Hence

$$
\operatorname{ind}_{F}(\Phi, U)=\operatorname{ind}_{F}(\bar{\Phi}, U)=\operatorname{ind}_{F}\left(\bar{\Phi}, U^{\prime}\right)=\operatorname{ind}_{F}\left(\Phi, U^{\prime}\right) .
$$

(iii) Similar to the above:

$$
\begin{aligned}
\operatorname{ind}_{F}(\Phi, U) & =\operatorname{ind}_{F}(\bar{\Phi}, U)=\operatorname{ind}_{F}\left(\bar{\Phi}, U_{1}\right)+\operatorname{ind}_{F}\left(\bar{\Phi}, U_{2}\right) \\
& =\operatorname{ind}_{F}\left(\Phi, U_{1}\right)+\operatorname{ind}_{F}\left(\Phi, U_{2}\right) .
\end{aligned}
$$

(iv) Without loss of generality we can assume that there exists a morphism $\Psi \in \mathcal{M}\left(\operatorname{cl} U \times I, E^{\prime}, v\right)\left(v: \operatorname{cl} U \times I \rightarrow E^{\prime}, v(\cdot, t)=\left.F\right|_{\mathrm{cl} U}\right)$ with an $F$-fundamental set $K$ (cf. 1.18) and such that $\Psi \circ i_{0}=\Phi$ and $\Psi \circ i_{1}=\Phi_{1}$. Let $(P, Q) \in \Psi$. Of course,

$$
\begin{aligned}
& \left(p_{0}, q_{0}\right)=\left(\left.P\right|_{P^{-1}(\operatorname{cl} U \times\{0\})},\left.Q\right|_{P^{-1}(\operatorname{cl} U \times\{0\})}\right) \in \Phi, \\
& \left(p_{1}, q_{1}\right)=\left(\left.P\right|_{P^{-1}(\operatorname{cl} U \times\{1\})},\left.Q\right|_{P^{-1}(\operatorname{cl} U \times\{1\})}\right) \in \Phi_{1} .
\end{aligned}
$$

Consider

$$
\left.Q\right|_{P^{-1}\left(F^{-1}(K) \cap \mathrm{cl} U\right)}: P^{-1}\left(F^{-1}(K) \cap \operatorname{cl} U\right) \rightarrow K
$$

and its extension $\bar{Q}: W \rightarrow K$. Then $(P, \bar{Q}) \in \mathcal{M}\left(\operatorname{cl} U \times I, E^{\prime}, v\right)$ is a compact homotopy between

$$
\left(p_{0}, \bar{q}_{0}\right)=\left(\left.P\right|_{P^{-1}(\operatorname{cl} U \times\{0\})},\left.\bar{Q}\right|_{P^{-1}(\operatorname{cl} U \times\{0\})}\right)
$$

and

$$
\left(p_{1}, \bar{q}_{1}\right)=\left(\left.P\right|_{P^{-1}(\operatorname{cl} U \times\{1\})},\left.\bar{Q}\right|_{P^{-1}(\operatorname{cl} U \times\{1\})}\right) .
$$

It is easily seen that $\left(p_{0}, \bar{q}_{0}\right)$ and $\left(p_{1}, \bar{q}_{1}\right)$ are compact pairs associated with $\left(p_{0}, q_{0}\right)$ and $\left(p_{1}, q_{1}\right)$, respectively. Hence

$$
\operatorname{ind}_{F}(\Phi, U)=\operatorname{ind}_{F}\left(\left[\left(p_{0}, \bar{q}_{0}\right)\right], U\right)=\operatorname{ind}_{F}\left(\left[\left(p_{1}, \bar{q}_{1}\right)\right], U\right)=\operatorname{ind}_{F}\left(\Phi_{1}, U\right) .
$$

(v) First, observe that $\left(\Phi^{\prime}, \operatorname{cl} U \cap T^{\prime}\right) \in \mathcal{M}^{F^{\prime}}\left(T^{\prime}, T\right)$ and if $K$ is an $F$-fundamental set for $\Phi$, then $K \cap T$ is an $F^{\prime}$-fundamental set for $\Phi^{\prime}$. Next, let $(p, q) \in \Phi$ and $(p, \bar{q})$ be its associated compact cotriad (with an $F$-fundamental set $K)$. Observe that if $p^{\prime}=\left.p\right|_{p^{-1}\left(\operatorname{cl} U \cap T^{\prime}\right)}$ and $q^{\prime}=$ $\left.q\right|_{p^{-1}\left(\operatorname{cl} U \cap T^{\prime}\right)}$, then $\left(p^{\prime}, q^{\prime}\right) \in \Phi^{\prime}$. Consider the cotriad $\left(p^{\prime}, r\right)$ such that $r=$ $\left.\bar{q}\right|_{p^{-1}\left(\mathrm{cl} U \cap T^{\prime}\right)}$. Observe that it is a compact cotriad associated with $\left(p^{\prime}, q^{\prime}\right)$. Indeed, $r$ is a compact extension of

$$
\left.q^{\prime}\right|_{p^{-1}\left(F^{\prime-1}(K \cap T) \cap \mathrm{cl} U \cap T^{\prime}\right)}: p^{-1}\left(F^{\prime-1}(K \cap T) \cap \operatorname{cl} U \cap T^{\prime}\right) \rightarrow K \cap T .
$$


Hence,

$$
\operatorname{ind}_{F}(\Phi, U)=\operatorname{ind}_{F}([(p, \bar{q})], U), \quad \operatorname{ind}_{F}\left(\left[\left(p^{\prime}, r\right)\right], U\right)=\operatorname{ind}_{F}\left(\Phi^{\prime}, U\right) .
$$

But from the restriction property of the generalized coincidence index for compact maps, $\operatorname{ind}_{F}([(p, \bar{q})], U)=\operatorname{ind}_{F}\left(\left[\left(p^{\prime}, r\right)\right], U\right)$, which ends the proof.

4. Applications. In this section we present conditions sufficient for the existence of solutions to an abstract inclusion

$$
A(x) \in \psi(x),
$$

where $A: E \rightarrow E^{\prime}$ is a Fredholm linear operator of nonnegative index $k$ ( $E$, $E^{\prime}$ are Banach spaces) and $\psi$ is a multivalued map. Next, we apply this result to a concrete boundary value problem in Banach spaces.

Of course, if $\psi$ is determined by an $A$-fundamentally contractible morphism $(\Psi, U)$ such that $\operatorname{ind}_{A}(\Psi, U)$ is not a trivial element of $\Pi_{k}$, then the inclusion (6) has a solution. But it is not simple to verify it when $E$ is not finite-dimensional.

Now we reformulate (6). Since $A$ is a Fredholm operator, there are two bounded linear projections $P: E \rightarrow E$ and $Q: E^{\prime} \rightarrow E^{\prime}$ such that $R(P)=\operatorname{Ker}(A)$ and $\operatorname{Ker}(Q)=R(A)$. Observe that $\operatorname{Ker}(P) \oplus \operatorname{Ker}(A)=E$ and $R(Q) \oplus R(A)=E^{\prime}$. Moreover, $\operatorname{dim} R(P), \operatorname{dim} R(Q)<\infty$ and $\operatorname{dim} R(P)-$ $\operatorname{dim} R(Q)=k$.

The restriction of $A$ to $\operatorname{Ker}(P)$ is a linear homeomorphism onto $R(A)$, hence it admits a right inverse $K_{P}: R(A) \rightarrow E$ defined by $K_{P}(y)=x$ if and only if $x \in \operatorname{Ker}(P)$ and $A(x)=y . K_{P}$ is a continuous linear map. Let $K_{P Q}: E^{\prime} \rightarrow E$ be the generalized inverse of $A$, i.e. $K_{P Q}=K_{P} \circ(I-Q)$. Clearly,

$$
R\left(K_{P Q}\right)=\operatorname{Ker}(P), \quad A \circ K_{P Q}=\operatorname{id}_{E^{\prime}}-Q, \quad K_{P Q} \circ A=\operatorname{id}_{E}-P .
$$

Denote by $J$ an arbitrary (but fixed) injective linear map from $R(Q)$ to $R(P)$. Of course, $J$ is continuous. Observe that $x \in \operatorname{cl} U$ is a solution to the inclusion (6) if and only if

$$
F(x) \in \phi(x),
$$

where

$$
F=\operatorname{id}_{E}-P: E \rightarrow \operatorname{Ker}(P) \oplus R(J)
$$

and

$$
\phi=\left(K_{P Q}+J \circ Q\right) \circ \psi(x): \operatorname{cl} U \multimap \operatorname{Ker}(P) \oplus R(J) .
$$

Indeed, if $y=A(x) \in \psi(x)$, then $Q(y)=Q \circ A(x)=0$ and $F(x)=$ $K_{P Q} \circ A(x)=\left(K_{P Q}+J \circ Q\right)(y) \in \phi(x)$. On the other hand, if $F(x) \in \phi(x)$, then $F(x)=\left(K_{P Q}+J \circ Q\right)(y)$, where $y \in \psi(x)$. It follows that $Q(y)=0$ and hence $A(x)=A \circ K_{P Q}(y)=y \in \psi(x)$. Moreover, $F$ is a Fredholm operator of index $k$ (equal to the index of $A$ ). 
Theorem 4.1. Assume that $U=E$ and

(i) ind $A=k \geq 0$;

(ii) there exists a metric space $W$ and a cotriad $E \stackrel{p}{\leftarrow} W \stackrel{q}{\rightarrow} E^{\prime}$ such that $\psi(x)=q\left(p^{-1}(x)\right),\left(\left[\left(\left.p\right|_{p^{-1}(\operatorname{cl} V)},\left.q\right|_{p^{-1}(\mathrm{cl} V)}\right)\right], V\right) \in \mathcal{M}^{A}\left(E, E^{\prime}\right)$ for every open bounded $V$, and some A-fundamental set for $\left(\left.p\right|_{p^{-1}(\mathrm{cl} V)},\left.q\right|_{p^{-1}(\mathrm{cl} V)}\right)$ contains 0 ;

(iii) there exists a constant $M>0$ such that for any $x \in E$, if $y \in$ $\left(\operatorname{id}_{E^{\prime}}-Q\right) \circ \psi(x)$, then $\|y\|_{E^{\prime}}<M$.

Additionally, if $R(Q) \neq 0$, assume that there exists a constant $R>0$ such that

(iv) if $\|P(x)\|_{E} \geq R$ and $\|x-P(x)\|_{E} \leq\left\|K_{P}\right\| M$, then $0 \notin Q \circ \psi(x)$;

(v) $\operatorname{ind}_{\mathcal{O}}\left(-\left.Q \circ \psi\right|_{B_{E}(0, R) \cap R(P)}, B_{E}(0, R) \cap R(P)\right) \in \Pi_{k}$ is nontrivial (here $\mathcal{O}: R(P) \rightarrow R(Q)$ is the zero operator $)$.

Then the inclusion (6) has a solution.

Proof. First, suppose that $R(Q) \neq 0$. If $x \in E$ is a solution to (6), then there is $y \in \psi(x)$ such that $Q(y)=0$ and $x-P(x)=K_{P Q}(y)$. Therefore, by (iii), $\|x-P(x)\|_{E}<\left\|K_{P}\right\| M$ and, by (iv), $\|P(x)\|_{E}<R$. Hence each solution $x$ is contained in the open bounded set $V=\{x \in$ $\left.E:\|x-P(x)\|_{E}<\left\|K_{P}\right\| M,\|P(x)\|_{E}<R\right\}$ and the map determined by $\left[\left(\left.p\right|_{p^{-1}(\mathrm{cl} V)},\left.q\right|_{p^{-1}(\mathrm{cl} V)}\right)\right]$ has no coincidence points with $A$ on bd $V$.

To simplify the notation put $p^{\prime}=\left.p\right|_{p^{-1}(\mathrm{cl} V)}$ and $q^{\prime}=\left.q\right|_{p^{-1}(\mathrm{cl} V)}$. Let $\left(p^{\prime}, \bar{q}^{\prime}\right)$ be a compact cotriad associated with $\left(p^{\prime}, q^{\prime}\right)$. Observe that

$$
K_{P Q}+J \circ Q: E^{\prime} \rightarrow \operatorname{Ker}(P) \oplus R(J)
$$

is a continuous linear isomorphism. By Remark 1.4 and Theorem 2.2, the morphisms $\left[\left(p^{\prime},\left(K_{P Q}+J \circ Q\right) \circ q^{\prime}\right)\right]$ and $\left[\left(p^{\prime},\left(K_{P Q}+J \circ Q\right) \circ \bar{q}^{\prime}\right)\right]$ are $F$ homotopic without coincidence points on bd $V$. Moreover, the morphisms $\left[\left(p^{\prime},\left(K_{P Q}+J \circ Q\right) \circ \bar{q}^{\prime}\right)\right]$ and $\left[\left(p^{\prime}, J \circ Q \circ \bar{q}^{\prime}\right)\right]$ are compactly $F$-homotopic (hence $F$-homotopic) without coincidence points on bd $V$. Indeed, consider the following homotopy $\mathrm{cl} V \times I \stackrel{R}{\leftarrow} W \times I \stackrel{S}{\rightarrow} \operatorname{Ker}(P) \oplus R(J)$ :

$$
R(w, \lambda)=\left(p^{\prime}(w), \lambda\right), \quad S(w, \lambda)=\left(\lambda K_{P Q}+J \circ Q\right) \circ \bar{q}^{\prime}(w) .
$$

Of course, it has no coincidence points with $F$ on bd $V$.

Hence, by the homotopy property of the index,

$$
\operatorname{ind}_{F}\left(\left[\left(p^{\prime},\left(K_{P Q}+J \circ Q\right) \circ \bar{q}^{\prime}\right)\right], V\right)=\operatorname{ind}_{F}\left(\left[\left(p^{\prime}, J \circ Q \circ \bar{q}^{\prime}\right)\right], V\right) .
$$

But if we put $\Phi=\left[\left(p^{\prime}, J \circ Q \circ \bar{q}^{\prime}\right)\right], T=R(J), T^{\prime}=F^{-1}(R(J))=R(P)$, $F^{\prime}=\left.F\right|_{T^{\prime}} \equiv 0$ and $\Phi^{\prime}=\left.\Phi\right|_{T^{\prime} \cap V}$, then, by the restriction property of the index and assumption $(\mathrm{v}), \operatorname{ind}_{F}\left(\left[\left(p^{\prime}, J \circ Q \circ \bar{q}^{\prime}\right)\right], V\right)$ is a nontrivial element of $\Pi_{k}$, because $\left.\bar{q}^{\prime}\right|_{\mathrm{cl} V \cap R(P)}=\left.q\right|_{\mathrm{cl} V \cap R(P)}$. Hence the inclusion $F(x) \in \phi(x)$ 
has a solution, which implies the existence of a solution to the inclusion $A(x) \in \psi(x)$.

If $Q \equiv 0$, then $K_{P Q}=K_{P}: E^{\prime} \rightarrow \operatorname{Ker}(P)$ is a continuous linear isomorphism and by assumption (iii) for all $y \in \psi(E),\|y\|_{E^{\prime}} \leq M$.

Let $V=\left\{x \in E:\|x\|_{E}<\left\|K_{P}\right\| M\right\}$. Observe that, by Remark 1.4, $\left(\left[\left(\left.p\right|_{p^{-1}(\mathrm{cl} V)},\left.K_{P} \circ q\right|_{p^{-1}(\mathrm{cl} V)}\right)\right], V\right) \in \mathcal{M}^{F}(E, E)$, because, as is easily seen, $\left(\left[\left(\left.p\right|_{p^{-1}(\mathrm{cl} V)},\left.q\right|_{p^{-1}(\mathrm{cl} V)}\right)\right], V\right) \in \mathcal{M}^{A}\left(E, E^{\prime}\right)$. Without loss of generality we can assume that a compact $F$-fundamental set of the multivalued map $\eta$, determined by the cotriad $\left(\left.p\right|_{p^{-1}(\mathrm{cl} V)},\left.K_{P} \circ q\right|_{p^{-1}(\mathrm{cl} V)}\right)$, is contained in the convex set $V$. Hence, its associated cotriad $\left(\left.p\right|_{p^{-1}(V)}, \bar{q}\right)$ determines a compact map $\bar{\eta}$ from $V$ to $V$ and by the Schauder Fixed Point Theorem there is $x \in V$ such that $x \in \bar{\eta}(x)$. Of course, then $x \in \eta(x)$ and hence $F(x) \in \eta(x)$. Finally, the inclusion $F(x) \in \phi(x)$ has a solution and the proof is complete.

REMARK 4.2. Observe that if $Q \not \equiv 0$ then instead of (iii) and (iv) in the above theorem we can assume that there is an open bounded $V \subset E$ such that there exists a compact $A$-fundamental set $K \subset E^{\prime}$ for $\left.\psi\right|_{V}, Q(\psi(V)) \subset$ $K$ and $F$ has no coincidence point with $\left.\left(\lambda K_{P Q}+J \circ Q\right) \circ \psi\right|_{\mathrm{cl} V}$ on bd $V$ for every $\lambda \in[0,1]$.

Indeed, it is easy to verify that then $K^{\prime}=\left(K_{P Q}+J \circ Q\right)(K) \supset J \circ$ $Q(\psi(V))$ and $K^{\prime}$ is an $F$-fundamental set for $\left(\lambda K_{P Q}+J \circ Q\right) \circ \psi$ for any $\lambda \in[0,1]$. Hence the morphisms $\left[\left(p^{\prime},\left(K_{P Q}+J \circ Q\right) \circ q^{\prime}\right)\right]$ and $\left[\left(p^{\prime}, J \circ Q \circ q^{\prime}\right)\right]$ are $F$-homotopic with an $F$-fundamental set $K^{\prime}$. The rest of the proof is identical.

If $Q \equiv 0$, instead of (iii) we can assume that there exists a bounded convex set $Z \subset \operatorname{Ker}(P)$ such that $K_{P} \circ \psi(Z) \subset Z$.

Below we illustrate the above result by a boundary value problem.

Let $E, E^{\prime}$ be Banach spaces, $J=[0, T] \subset \mathbb{R}, \chi, \chi^{\prime}$ be the Hausdorff measures of noncompactness on $E$ and $E^{\prime}$ respectively, and let $\xi$ be the Hausdorff measure of noncompactness on the space $C=C(J, E)$ of continuous functions from $J$ to $E$.

Recall that if $\mathcal{B}$ is the family of all bounded subsets of $E$, then the Hausdorff measure of noncompactness $\chi: \mathcal{B} \rightarrow[0, \infty)$ is given by the formula $\chi(B)=\inf \left\{r>0: B\right.$ has a finite $r$-net $\left.\left({ }^{4}\right)\right\}$.

Let $Z$ be the set of all positive numbers $k$ such that the Fredholm linear operator $D: E \rightarrow E^{\prime}$ is a $\left(k, \chi, \chi^{\prime}\right)$-set contraction $\left({ }^{5}\right)$. Following [1] we define

\footnotetext{
$\left({ }^{4}\right)$ That is, a finite number of points $x_{1}, \ldots, x_{k} \in E$ such that $B \subset \bigcup_{i=1}^{k} B_{E}\left(x_{i}, r\right)$.

$\left({ }^{5}\right)$ That is, for any bounded set $B \subset E$, the set $D(B)$ is bounded and $\chi^{\prime}(D(B)) \leq$ $k \chi(B)$.
} 


$$
\|D\|^{\left(\chi, \chi^{\prime}\right)}:=\inf Z .
$$

Observe that $\|D\|^{\left(\chi, \chi^{\prime}\right)} \leq\|D\|$.

Let $g: J \times E \rightarrow E$ and $h: J \times E \times E \rightarrow E$ be continuous (singlevalued) maps such that

$\left(f_{1}\right) \quad g$ is completely continuous and uniformly continuous on bounded sets,

$\left(f_{2}\right) \quad$ there exists a continuous function $\varrho: J \rightarrow[0,1)$ such that

$$
\left\|h\left(t, u_{1}, v_{1}\right)-h\left(t, u_{2}, v_{2}\right)\right\|_{E} \leq \varrho(t)\left(\left\|u_{1}-u_{2}\right\|_{E}+\left\|v_{1}-v_{2}\right\|_{E}\right)
$$

for any $t \in J, u_{1}, u_{2}, v_{1}, v_{2} \in E$.

Suppose that $g, h$ have sublinear growth, i.e.

$\left(f_{3}\right) \quad$ there exist continuous functions $m, n: J \rightarrow[0, \infty)$ such that

$$
\|g(t, u)+h(t, u, v)\|_{E} \leq m(t)+n(t)\|u\|_{E} \quad \text { for }(t, u, v) \in J \times E \times E .
$$

Consider the following boundary value problem:

$$
\left\{\begin{array}{l}
\left.u^{\prime}(t)=f\left(t, u(t), u^{\prime}(t)\right)\right), \\
L_{1}(u(0))+L_{2}(u(T))=b(u(0)),
\end{array}\right.
$$

where $f(t, u, v)=g(t, u)+h(t, u, v), b: E \rightarrow E^{\prime}$ is a compact continuous map and $L_{1}, L_{2}: E \rightarrow E^{\prime}$ are linear operators such that $L=L_{1}+L_{2}$ is a Fredholm operator of nonnegative index.

We can rewrite problem (7) as follows:

$$
A(z, y)=\psi(z, y)
$$

where $A, \psi: E \times C \rightarrow E^{\prime} \times C$ and

$$
\begin{aligned}
& A(z, y)=(L(z), y) \\
& \psi(z, y)=\left(b(z)-L_{2}\left(\int_{0}^{T} y(s) d s\right), f\left(\cdot, z+\int_{0}^{1} y(s) d s, y(\cdot)\right)\right) .
\end{aligned}
$$

It is easily seen that if $(z, y)$ is a solution of (8), then $u$ defined by $u(t)=$ $z+\int_{0}^{t} y(s) d s$ is a solution of (7).

As usual we equip the spaces $E \times C$ and $E^{\prime} \times C$ with the max-norms, i.e. for $z \in E, c \in E^{\prime}$ and $y \in C,\|(z, y)\|_{1}=\max \left(\|z\|_{E},\|y\|_{C}\right),\|(c, y)\|_{2}=$ $\max \left(\|c\|_{E^{\prime}},\|y\|_{C}\right)$. Denote the Hausdorff measures of noncompactness in $E \times C$ and $E^{\prime} \times C$ by $\mu$ and $\mu^{\prime}$, respectively. Let $\pi_{E}, \pi_{C}$ (resp. $\left.\pi_{E^{\prime}}, \pi_{C}^{\prime}\right)$ be projections of $E \times C$ (resp. $E^{\prime} \times C$ ) onto $E$ and $C$ (resp. onto $E^{\prime}$ and $C$ ). Observe that if $S$ is a bounded subset of $E \times C$, then $\mu(S)=$ $\max \left(\chi\left(\pi_{E}(S)\right), \xi\left(\pi_{C}(S)\right)\right)$.

Denote by $P_{L}, Q_{L}, K_{P_{L}}$ the respective projections and the right inverse for $L$. Moreover, let $M=\sup _{t \in J} m(t), N=\sup _{t \in J} n(t), N_{1}=\sup _{t \in J} t n(t)$. 
Theorem 4.3. Assume that $f, b, L$ are as above, $R\left(L_{2}\right) \subset R(L), Q_{L} \not \equiv 0$ and additionally:

$\left(f_{4}\right) \sup _{t \in J}(\varrho(t)(t+1))<1$ and $\left\|K_{P_{L}}\right\|^{\left(\chi, \chi^{\prime}\right)}<\frac{1-\sup _{t \in J} \varrho(t)(t+1)}{\sup _{t \in J}(\varrho(t))}$,

$\left(f_{5}\right) \quad\left\|L_{2}\right\| \cdot T<1$

$\left(f_{6}\right) \quad N e^{N_{1}}<1$,

$\left(f_{7}\right) \quad$ there exists $R>0$ such that for all $z$ if $\|P(z)\|_{E}>R$, then $Q_{L}(b(z))$ $\neq 0$ and $\operatorname{ind}_{\mathcal{O}}\left(Q_{L} \circ b, B(0, R) \cap R(P)\right)$ is a nontrivial element of $\Pi_{k}$.

Then problem (7) has a solution.

P r o of. We prove that problem (8), equivalent to (7), has a solution.

Observe that $A$ is a Fredholm linear operator and its index is equal to the index of $L$.

STEP 1. We prove that if $V$ is an open bounded subset of $E \times C$,

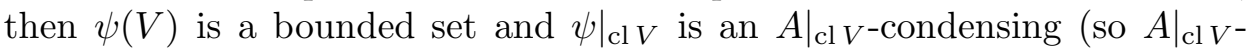
fundamentally contractible) map.

Take an arbitrary subset $S$ of $V$. We have to prove that $\mu^{\prime}(\psi(S))<$ $\mu^{\prime}(A(S))$. Let $\chi\left(\pi_{E}(S)\right)=\varepsilon$ and $\xi\left(\pi_{C}(S)\right)=\delta$. Then

$$
\mu^{\prime}(A(S))=\max \left(\chi^{\prime}\left(\pi_{E^{\prime}}(A(S))\right), \xi\left(\pi_{C}(A(S))\right)\right)=\max \left(\chi^{\prime}\left(\pi_{E^{\prime}}(A(S))\right), \delta\right) .
$$

Observe that, since $\operatorname{Ker}(L)=R\left(P_{L}\right)$ is of finite dimension,

$$
\chi\left(\pi_{E}(S)\right)=\chi\left(\left(\operatorname{id}_{E}-P_{L}\right) \circ \pi_{E}(S)\right)=\chi\left(K_{P_{L}} \circ L \circ \pi_{E}(S)\right) .
$$

But

$$
\chi\left(K_{P_{L}} \circ L \circ \pi_{E}(S)\right) \leq\left\|K_{P_{L}}\right\|^{\left(\chi, \chi^{\prime}\right)} \chi^{\prime}\left(L \circ \pi_{E}(S)\right)
$$

and $L\left(\pi_{E}(S)\right)=\pi_{E^{\prime}}(A(S))$, hence

$$
\chi^{\prime}\left(\pi_{E^{\prime}}(A(S))\right) \geq \frac{\chi\left(\pi_{E}(S)\right)}{\left\|K_{P_{L}}\right\|\left(\chi, \chi^{\prime}\right)}=\frac{\varepsilon}{\left\|K_{P_{L}}\right\|\left(\chi, \chi^{\prime}\right)} .
$$

Finally we get

$$
\mu^{\prime}(A(S)) \geq \max \left(\frac{\chi\left(\pi_{E}(S)\right)}{\left\|K_{P_{L}}\right\|\left(\chi, \chi^{\prime}\right)}, \delta\right) .
$$

Now we estimate $\mu(\psi(S))$. Obviously,

$$
\begin{aligned}
& \mu^{\prime}(\psi(S))=\max \left(\chi^{\prime}\left(\left\{b(z)-L_{2}\left(\int_{0}^{T} y(s) d s\right):(z, y) \in S\right\}\right),\right. \\
&\left.\xi\left(\left\{f\left(\cdot, z+\int_{0}^{1} y(s) d s, y(\cdot)\right):(z, y) \in S\right\}\right)\right) .
\end{aligned}
$$


Observe that

$$
\begin{aligned}
& \chi^{\prime}\left(\left\{b(z)-L_{2}\left(\int_{0}^{T} y(s) d s\right):(z, y)\right.\right.\in S\}) \\
& \leq \chi^{\prime}\left(\left\{L_{2}\left(\int_{0}^{T} y(s) d s\right): y \in \pi_{C}(S)\right\}\right),
\end{aligned}
$$

because $\chi^{\prime}\left(\left\{b(z): z \in \pi_{E}(S)\right\}\right)=0$. Now, for arbitrary $\delta_{1}>0$, take an element $\bar{y}$ from a $\left(\delta+\delta_{1}\right)$-net of $\pi_{C}(S)$. If $\|\bar{y}-y\|_{C}<\delta+\delta_{1}$, then

$$
\begin{aligned}
\left\|L_{2}\left(\int_{0}^{T} \bar{y}(s) d s\right)-L_{2}\left(\int_{0}^{T} y(s) d s\right)\right\|_{E} & \\
& \leq\left\|L_{2}\right\| \int_{0}^{T}\|\bar{y}(s)-y(s)\|_{E} d s<\left\|L_{2}\right\| T\left(\delta+\delta_{1}\right) .
\end{aligned}
$$

Hence $\chi^{\prime}\left(\left\{L_{2}\left(\int_{0}^{T} y(s) d s\right): y \in \pi_{L}(S)\right\}\right) \leq\left\|L_{2}\right\| T \delta<\delta$.

Consider the family of functions

$$
F_{1}(S)=\left\{g_{z, y} \in C: g_{z, y}(t)=g\left(t, z+\int_{0}^{t} y(s) d s\right), \quad(z, y) \in S\right\} .
$$

Since $S$ is bounded and $g$ is completely continuous, the sets $\left\{g_{z, y}(t):(z, y)\right.$ $\in S\}$ are compact for any $t \in J$. Moreover, the family $F_{1}(S)$ is uniformly equicontinuous. Hence, by Ascoli's Theorem (cf. [11]), $\xi\left(F_{1}(S)\right)=0$. This gives

$$
\begin{aligned}
\xi\left(\left\{f\left(\cdot, z+\int_{0}^{1} y(s) d s, y(\cdot)\right)\right.\right. & :(z, y) \in S\}) \\
\leq & \xi\left(\left\{h\left(\cdot, z+\int_{0}^{1} y(s) d s, y(\cdot)\right):(z, y) \in S\right\}\right) .
\end{aligned}
$$

If we take arbitrary $\varepsilon_{1}>0$ and $\delta_{1}>0, \bar{z}$ from an $\left(\varepsilon+\varepsilon_{1}\right)$-net of $\pi_{E}(S), \bar{y}$ from a $\left(\delta+\delta_{1}\right)$-net of $\pi_{C}(S)$ and if $\|\bar{z}-z\|_{E}<\varepsilon+\varepsilon_{1}$ and $\|\bar{y}-y\|_{C}<\delta+\delta_{1}$, then

$$
\begin{aligned}
\| h(t & \left., \bar{z}+\int_{0}^{t} \bar{y}(s) d s, y(t)\right)-h\left(t, z+\int_{0}^{t} y(s) d s, y(t)\right) \|_{E} \\
& \leq \varrho(t)\left(\|\bar{z}-z\|_{E}+\int_{0}^{t}\|\bar{y}(s)-y(s)\|_{E} d s+\|\bar{y}(t)-y(t)\|_{E}\right) \\
& <\varrho(t)\left(\varepsilon+\varepsilon_{1}+t\left(\delta+\delta_{1}\right)+\delta+\delta_{1}\right) \leq \sup _{t \in J} \varrho(t)\left(\varepsilon+\varepsilon_{1}+(t+1)\left(\delta+\delta_{1}\right)\right) .
\end{aligned}
$$

Therefore $\xi\left(\pi_{C}^{\prime}(\psi(S))\right) \leq \sup _{t \in J} \varrho(t)(\varepsilon+(t+1) \delta)$. 
Now, it is easy to check that assumptions $\left(f_{4}\right)-\left(f_{5}\right)$ imply that $\mu^{\prime}(\psi(S))$ $<\mu^{\prime}(A(S))$.

STEP 2. Since $b$ is a compact map, there exists $Z>0$ such that $b(E) \subset$ $B_{E^{\prime}}(0, Z)$. Denote by $P, Q, K_{P}$ and $K_{P Q}$ the respective projections, the right inverse and the generalized inverse for $A$ (see the beginning of this section).

Now, we introduce a set $V$ satisfying the assumptions of Remark 4.2. Let

$$
\begin{aligned}
V=\{(z, y) \in E \times C: z= & z_{0}+z_{1}, z_{0} \in B_{E}(0, R) \cap R\left(P_{L}\right), \\
& \left.z_{1} \in B_{E}\left(0, R_{1}\right) \cap \operatorname{Ker}\left(P_{L}\right), y \in B_{C}\left(0, R_{2}\right)\right\},
\end{aligned}
$$

where $R$ is as in assumption $\left(f_{7}\right)$,

$$
R_{1}>\frac{\left\|K_{P_{L}}\right\|\left(Z+M e^{N_{1}}+N R e^{N_{1}}\right)}{1-N e^{N_{1}}}, \quad R_{2}=\left(M+N\left(R+R_{1}\right)\right) e^{N_{1}} .
$$

We can find a compact $A$-fundamental set for $\left.\psi\right|_{\mathrm{cl} V}$ containing $Q \circ \psi(V)$ (since it is a bounded subset of the finite-dimensional space $R(Q)$, see Example 1.10).

We will show that there is no solution of the problem $F(z, y)=\left(\lambda K_{P Q}+\right.$ $J \circ Q) \circ \psi(z, y)$ on the boundary of the set $V$ for every $\lambda \in[0,1]$.

Assume that $(z, y) \in \operatorname{cl} V$ is such a solution for some $\lambda \in[0,1]$. Let $z=z_{0}+z_{1}$ where $z_{0} \in R\left(P_{L}\right), z_{1} \in \operatorname{Ker}\left(P_{L}\right)$. Then

$$
\begin{aligned}
\left(z_{1}, y\right)=\left(\lambda K_{P_{L}} \circ\left(\operatorname{id}_{E^{\prime}}-Q_{L}\right) \circ b(z)-\lambda K_{P_{L}} \circ L_{2}\left(\int_{0}^{T} y(s) d s\right),\right. \\
\left.f\left(\cdot, z+\int_{0}^{1} y(s) d s, y(\cdot)\right)\right)+J\left(Q_{L} \circ b(z), 0\right) .
\end{aligned}
$$

Hence

$$
\begin{gathered}
Q(b(z))=0 \\
z_{1}=\lambda K_{P_{L}}\left(\operatorname{id}_{E^{\prime}}-Q\right)(b(z))-\lambda K_{P_{L}}\left(L_{2}\left(\int_{0}^{T} y(s) d s\right)\right), \\
y=\left(\cdot, z+\int_{0} y(s) d s, y(\cdot)\right) .
\end{gathered}
$$

Equality (9) and assumption $\left(f_{7}\right)$ imply at once $\left\|z_{0}\right\|<R$. Moreover, by (11),

$$
\begin{aligned}
\|y(t)\|_{E} & =\left\|f\left(t, z_{0}+z_{1}+\int_{0}^{t} y(s) d s, y(t)\right)\right\|_{E} \\
& \leq m(t)+n(t)\left(\left\|z_{0}\right\|_{E}+\left\|z_{1}\right\|_{E}+\int_{0}^{t}\|y(s)\|_{E} d s\right)
\end{aligned}
$$


and by the Gronwall inequality

$$
\|y(t)\|_{E} \leq\left(m(t)+n(t)\left(\left\|z_{0}\right\|_{E}+\left\|z_{1}\right\|_{E}\right)\right) e^{N_{1}} .
$$

Hence assumption $\left(f_{6}\right)$ gives

$\|y\|_{C}=\sup _{t \in J}\|y(t)\|_{E} \leq\left(M+N\left(\left\|z_{0}\right\|_{E}+R_{1}\right)\right) e^{N_{1}}<\left(M+N\left(R+R_{1}\right)\right) e^{N_{1}}=R_{2}$.

Finally, if $\lambda=0$, then (10) gives $\left\|z_{1}\right\|_{E}=0<R_{1}$. If $\lambda \in(0,1]$, then by (10) and assumption $\left(f_{5}\right)$,

$$
\left\|z_{1}\right\|_{E} \leq \lambda\left\|K_{P_{L}}\right\|\left(Z+\|y\|_{C}\right)<\left\|K_{P_{L}}\right\|\left(Z+\left(M+N\left(R+R_{1}\right)\right) e^{N_{1}}\right)<R_{1} .
$$

SteP 3. Observe that assumption (v) of Theorem 4.1 is also satisfied. Indeed, assumption $\left(f_{7}\right)$ implies the nontriviality of the index, because

$$
\left.Q \circ \psi\right|_{B(0, R) \cap R(P)}(z, y)=Q(b(z), 0)=Q_{L}(b(z)) .
$$

Now, by Theorem 4.1 with Remark 4.2 the proof is complete.

REMARK 4.4. If $Q_{L} \equiv 0$ then we also get an existence result for problem (7) if we replace assumptions $\left(f_{6}\right)$ and $\left(f_{7}\right)$ by

$$
\left\|K_{P_{L}}\right\| \cdot\left\|L_{2}\right\|+T N<1 .
$$

Indeed, one can easily verify that the set

$$
Z=\left\{(z, y):\left\|z+\int_{0}^{t} y(s) d s\right\|<R^{\prime}, \forall t\right\} \cap\left\{(z, y):\|y\|_{C}<R^{\prime \prime}\right\} \cap \operatorname{Ker}(P),
$$

where

$$
R^{\prime}>\frac{\left\|K_{P_{L}}\right\| S+T M}{1-\left\|K_{P_{L}}\right\| \cdot\left\|L_{2}\right\|-T N} \quad \text { and } \quad R^{\prime \prime}>M+N R^{\prime}
$$

satisfies Remark 4.2.

\section{References}

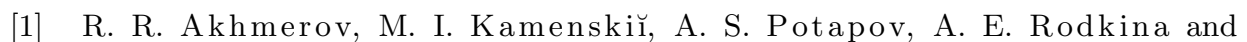
B. N. Sadovskiur, Measures of Noncompactness and Condensing Operators, Birkhäuser, Basel, 1992.

[2] S. Armentrout and T. M. Price, Decomposition into compact sets with UV properties, Trans. Amer. Math. Soc. 141 (1969), 433-442.

[3] Yu. G. Borisovich, B. D. Gel'man, A. D. Myshkis and V. V. Obukhovskiŭ, Topological methods in the fixed point theory of multivalued mappings, Russian Math. Surveys 35 (1980), 65-143.

[4] Yu. G. Borisovich, V. G. Zvyagin and Yu. I. Sapronov, Nonlinear Fredholm mappings and Leray-Schauder theory, ibid. 32 (1977), 3-54.

[5] K. G,eba, Algebraic topology methods in the theory of compact fields in Banach spaces, Fund. Math. 46 (1964), 177-209. 
[6] K. G, eba, I. Massabò and A. Vignoli, Generalized topological degree and bifurcation, in: Nonlinear Functional Analysis and its Applications (Maratea, 1985), Reidel, 1986, 55-73.

[7] S. Goldberg, Unbounded Linear Operators. Theory and Applications, McGrawHill, 1966.

[8] L. G'orniewicz, Homological methods in fixed-point theory of multi-valued maps, Dissertationes Math. 129 (1976).

[9] L. G'orniewicz and W. Kryszewski, Bifurcation invariants for acyclic mappings, Rep. Math. Phys. 31 (1992), 217-239.

[10] J. Izé, I. Massabò and A. Vignoli, Degree theory for equivariant maps I, Trans. Amer. Math. Soc. 315 (1989), 433-510.

[11] J. L. Kelley, General Topology, Van Nostrand, New York, 1955.

[12] W. Kryszewski, Homotopy properties of set-valued mappings, Wyd. Uniwersytetu Mikołaja Kopernika, Toruń, 1997.

[13] - Topological and approximation methods in the degree theory of set-valued maps, Dissertationes Math. 336 (1994).

[14] - , The fixed-point index for the class of compositions of acyclic set-valued maps on ANR's, Bull. Sci. Math. 120 (1996), 129-151.

[15] - Remarks to the Vietoris Theorem, Topol. Methods Nonlinear Anal. 8 (1996), 383-405.

[16] R. C. Lacher, Cell-like mappings and their generalizations, Bull. Amer. Math. Soc. 83 (1977), 336-552.

[17] J. Mawhin, Topological Degree Methods in Nonlinear Boundary Value Problems, CBMS Regional Conf. Ser. in Math. 40, Amer. Math. Soc., Providence, 1979.

[18] T. Pruszko, Some applications of the topological degree theory to multi-valued boundary value problems, Dissertationes Math. 229 (1984).

Faculty of Mathematics and Computer Science

Nicholas Copernicus University

Chopina 12/18

87-100 Torun, Poland

E-mail: dgabor@mat.uni.torun.pl 